\title{
The temperature-dependent conformational ensemble of SARS-CoV-2 main protease $\left(\mathrm{M}^{\text {pro}}\right)$
}

\author{
Ali Ebrahim ${ }^{*, 1,2}$, Blake T. Riley ${ }^{*, 2}$, Desigan Kumaran³, Babak Andi4,5, Martin R. Fuchs ${ }^{4}$, Sean McSweeney ${ }^{4,5}$, \\ Daniel A. Keedy $\#, 2,6,7$
}

${ }^{I}$ Diamond Light Source, Harwell Science and Innovation Campus, Didcot OX11 ODE, England, ${ }^{2}$ Structural Biology Initiative, CUNY Advanced Science Research Center, New York, NY 10031, ${ }^{3}$ Biology Department, Brookhaven National Laboratory, Upton, NY 11973 ,

${ }^{4}$ National Synchrotron Light Source II, Brookhaven National Laboratory, Upton, NY 11973, ${ }^{5}$ National Virtual Biotechnology

Laboratory (NVBL), US Department of Energy, Washington, DC, United States, ${ }^{6}$ Department of Chemistry and Biochemistry, City

College of New York, New York, NY 10031, ' Ph.D. Programs in Biochemistry, Biology, and Chemistry, The Graduate Center-City

University of New York, New York, NY 10016, *contributed equally, " speaker \& corresponding author

\section{dkeedy@gc.cuny.edu}

The COVID-19 pandemic, instigated by the SARS-CoV-2 coronavirus, continues to plague the globe. The SARS-CoV-2 main protease, or $\mathrm{M}^{\text {pro }}$, is a promising target for development of novel antiviral therapeutics. Previous X-ray crystal structures of $\mathrm{M}^{\text {pro }}$ were obtained at cryogenic temperature or room temperature only. Here we report a series of high-resolution crystal structures of unliganded $\mathrm{M}^{\text {pro }}$ across multiple temperatures from cryogenic to physiological, and another at high humidity. We interrogate these datasets with parsimonious multiconformer models, multi-copy ensemble models, and isomorphous difference density maps. Our analysis reveals a temperature-dependent conformational landscape for $\mathrm{M}^{\text {pro }}$, including a mobile water interleaved between the catalytic dyad, mercurial conformational heterogeneity in a key substrate-binding loop, and a far-reaching intramolecular network bridging the active site and dimer interface. Our results may inspire new strategies for antiviral drug development to counter-punch COVID-19 and combat future coronavirus pandemics.
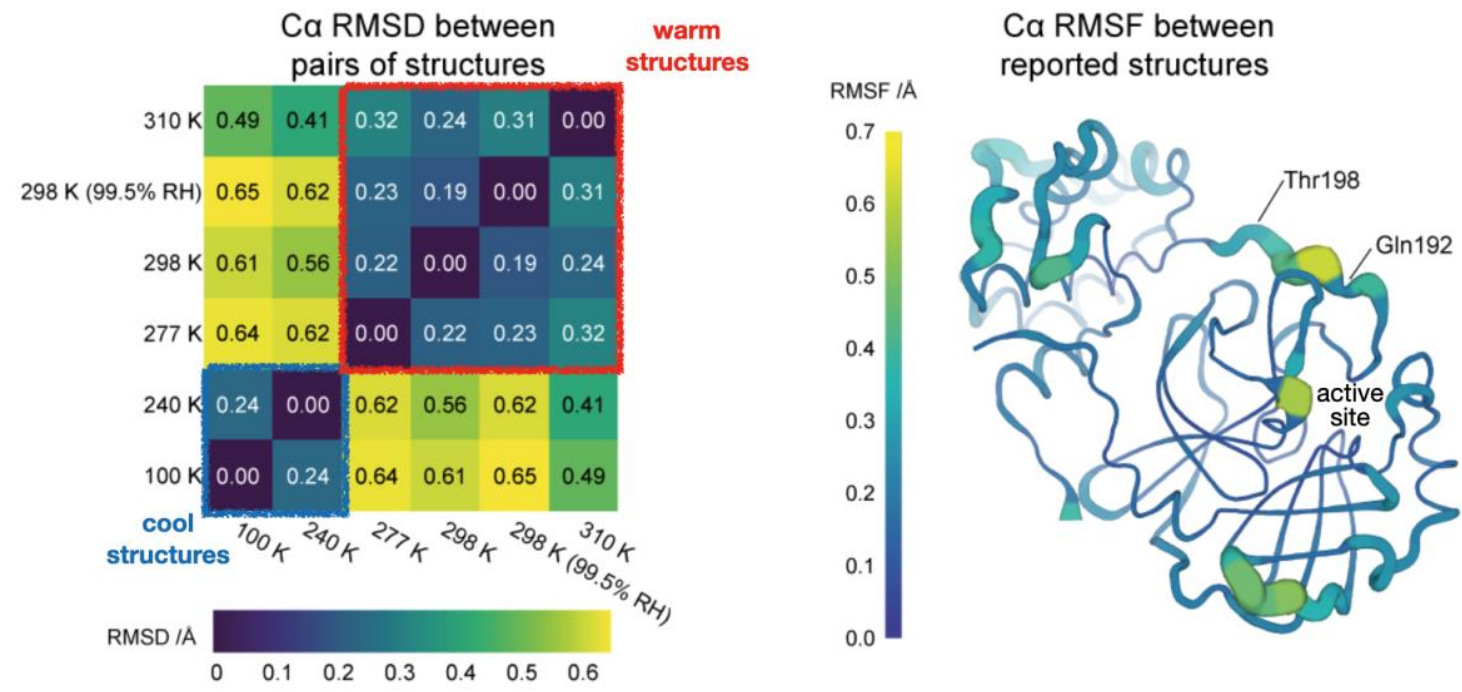

Figure 1. Conformational variability in SARS-CoV-2 $\mathrm{M}^{\text {pro }}$ as a function of temperature. Left: Heatmap of global C $\alpha$ atom root-meansquare deviation (RMSD) between pairs of structures, revealing temperature-dependent clustering. Right: Cartoon putty representation of local root-mean-square fluctuations (RMSF) among all structures, highlighting flexibility not only at the active site but also elsewhere in the enzyme.

Keywords: SARS-CoV-2, X-ray crystallography, multitemperature crystallography, protein structure, protein flexibility

This research was supported by the DOE Office of Science through the National Virtual Biotechnology Laboratory (NVBL) with funding from the Coronavirus CARES Act and additional funding was provided by Brookhaven National Laboratory (BNL) for research on COVID-19 (LDRD 20-042). 